\title{
Optimal Acupuncture Methods for Nonspecific Low Back Pain: A Systematic Review and Bayesian Network Meta-Analysis of Randomized Controlled Trials
}

\author{
Linjia Wang (D' \\ Zihan Yin' \\ Yutong Zhang' \\ Mingsheng Sun' \\ Yang Yu' \\ Yanming $\operatorname{Lin}^{2}$ \\ Ling Zhao'
}

'School of Acu-Mox and Tuina, Chengdu University of Traditional Chinese Medicine, Chengdu, 610075, People's Republic of China; ${ }^{2} \mathrm{Hospital}$ of Chengdu University of Traditional Chinese Medicine, Chengdu, 610075, Sichuan, People's Republic of China
Correspondence: Ling Zhao; Yanming Lin Email zhaoling@cdutcm.edu.cn; linyanming@cdutcm.edu.cn
Background: Nonspecific low back pain (NLBP) is a common disabling disease that cannot be attributed to a specific, recognizable pathology. The use of acupuncture for NLBP is supported by several guidelines and systematic reviews. However, the efficacy of different acupuncture methods for NLBP management is still debated. This study ranked the effectiveness of acupuncture methods using network meta-analysis to screen out the optimal acupuncture methods and expound the current controversies for their effective application in health policies as well as guiding clinical operations.

Methods: The following databases were searched for relevant randomized controlled trials (RCTs) from inception to December 20, 2020: China National Knowledge Infrastructure, VIP Database for Chinese Technical Periodicals, WANFANG Database, Chinese biomedical literature service system, PubMed, Web of Science, Embase, and Cochrane Library. Relevant registration platforms, including the International Standard Randomised Controlled Trial Number Register (ISRCTN) and Chinese Clinical Trial Registry (ChiCTR), were also searched. Manual retrieval and tracking of references was also performed. Pairwise meta-analysis and Bayesian network meta-analysis using Revman and ADDIS, respectively, were performed and standardized mean differences examined. The primary outcome was visual analog scale (VAS) score and the secondary outcome was Oswestry Disability Index (ODI) score. Safety was defined as the incidence of adverse events.

Results: A total of 30 trials with 3196 participants were analyzed; 16.67\% of which showed a high risk of bias. The results indicated that fire acupuncture plus manual acupuncture, auricular needling, and electroacupuncture plus warm acupuncture were most effective in reducing VAS score. The most effective interventions for reducing ODI score were manual acupuncture plus conventional medicines, followed by moxibustion and manual acupuncture plus moxibustion. Manual acupuncture plus moxibustion was dominant in the cluster ranking. Acupuncture showed a lower incidence of adverse events $(7.70 \%)$ than other interventions (conventional medicines, routine care, and placebo; $12.24 \%)$.

Conclusion: We found that manual acupuncture plus moxibustion is the most effective way to reduce NLBP pain and disability. Acupuncture is safer than other interventions. However, more direct comparative evidence from high-quality, large-sample, multicenter RCTs is needed to validate these findings.

Keywords: acupuncture, nonspecific low back pain, network meta-analysis 


\section{Background}

Nonspecific low back pain (NLBP) is a type of low back pain (LBP) that cannot be attributed to a specific, recognizable pathology (such as infection, tumor, osteoporosis, fracture, structural malformation, nerve root syndrome, or cauda equina syndrome). ${ }^{1}$ Of patients with LBP, $90 \%$ to 95\% have NLBP. ${ }^{2,3}$ By definition, NLBP has no known pathoanatomical cause, because current radiological methods cannot provide a definitive diagnosis. ${ }^{4,5}$ Despite this, NLBP is one of the most common disabling diseases in the world, ${ }^{6,7}$ owing to its typical intermittency and periodicity. ${ }^{8}$ Some studies have shown that as many as two-thirds of adults have LBP at some time,,${ }^{9,10}$ and in both developed and developing countries, LBP is the leading cause of years lived with disability and ranks sixth in overall disease burden. ${ }^{11,12}$

Regarding NLBP treatment, one review study ${ }^{13}$ found that paracetamol and nonsteroidal anti-inflammatory preparations (NSAIDs) are recommended treatments; other studies $^{14,15}$ suggest that skeletal muscle relaxants and opioid analgesics are the most common drugs for NLBP. However, paracetamol is ineffective in patients with acute LBP $^{16}$ and some researchers ${ }^{17,18}$ have claimed that NSAIDs and opioid analgesics may cause serious side effects, such as falls, fractures, depression, sexual dysfunction, ${ }^{19}$ and even dependence and overdose deaths. ${ }^{20}$ Based on this, the US Centers for Disease Control and Prevention guideline ${ }^{21}$ recommends nonpharmacologic therapy and nonopioid pharmacologic therapy as treatments for chronic pain. Additionally, some researchers have suggested that nonpharmacological therapies are more important in the treatment of persistent LBP. $^{22}$ Therefore, the use of alternative medicine to treat NLBP is receiving increased attention.

Acupuncture originated in ancient China, and is one of the most important nonpharmacological therapies. Numerous studies ${ }^{23,24}$ have reported that acupuncture is effective for NLBP. Recently, a Cochrane systematic review $^{25}$ assessed the effects of acupuncture treatment on NLBP. However, the review mainly focused on acupuncture compared with sham intervention, no treatment, and usual care and did not include other acupuncture methods, such as moxibustion and electroacupuncture. In addition, head-to-head randomized controlled trials (RCTs) have mainly confirmed the efficacy of acupuncture by comparing it with conventional medicines and placebos, rather than comparing the efficacy of different acupuncture methods (eg, single or combined interventions). Therefore, it is necessary to identify the optimal acupuncture methods for NLBP treatment. In this study, we used network meta-analysis (NMA) to investigate this question. NMA can combine direct and indirect evidence from any intervention to form an RCT network to compare different treatment options. Using this method, we ranked the priority of acupuncture methods using NMA to screen out the optimal acupuncture methods and expound the current controversies for their effective application in health policies as well as guiding clinical operations.

\section{Methods}

The study design followed the PRISMA-NMA guidelines ${ }^{26}$ and a version of this study was registered on Open Science Framework (Registration DOI: 10.17605/ OSF.IO/TKDQ).

\section{Search Strategy}

To obtain a sufficient number of articles, we searched major databases and clinical trial registries in both Chinese and English from inception to December 20, 2020. The search time was December 20, 2020. The databases were China National Knowledge Infrastructure (CNKI), VIP Database for Chinese Technical Periodicals (VIP), WANFANG Database (WF), Chinese biomedical literature service system (SinoMed), PubMed, Web of Science (WOS), Embase, and Cochrane Library. The clinical trial registries were the International Standard Randomised Controlled Trial Number Register (ISRCTN) and the Chinese Clinical Trial Registry (ChiCTR). We used the following combination of MeSH terms and free words to search the literature: (1) low back pain, lumbago, low back ache; (2) acupuncture, moxibustion, electroacupuncture, fire acupuncture, warm acupuncture, needle warming moxibustion, heat sensitive moxibustion, auricular needling, acupoint, etc.; (3) randomized controlled trial, controlled clinical trial, etc. Table 1 shows the full PubMed search strategy; we used similar retrieval methods for the other databases. Subsequently, we used manual retrieval and tracking of references. We manually searched articles in Chinese Acupuncture and Moxibustion, Acupuncture Research, and the Shanghai Journal of Acupuncture and Moxibustion from their inception to December 2020. In addition, we also searched some references of relevant studies, such as the study of $\mathrm{Mu}^{25}$ The 
Table I Search Strategy (PubMed)

\begin{tabular}{|c|c|}
\hline Steps & Search \\
\hline$\# 1$ & $\begin{array}{l}\text { “low back pain”[MeSH] OR "lumbago"[Ti/Ab] OR “low } \\
\text { back ache" }[\mathrm{Ti} / \mathrm{Ab}]\end{array}$ \\
\hline$\# 2$ & 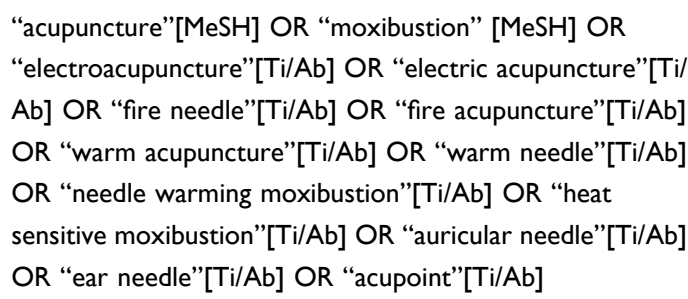 \\
\hline \#3 & $\begin{array}{l}\text { “randomized controlled trial”[Pt] OR “controlled clinical } \\
\text { trial”[Pt] OR “randomized”[Ti/Ab] OR “clinical trials as } \\
\text { topic”[MeSH] OR “randomly"[Ti/Ab] OR “trial”[Ti] OR } \\
\text { “clinical”[Ti] }\end{array}$ \\
\hline$\# 4$ & English[Lan] \\
\hline$\# 5$ & $\#$ I AND \#2 AND \#3 AND \#4 \\
\hline
\end{tabular}

search strategy was carried out by an expert with experience in the evidence-based medicine field.

\section{Criteria for Study Inclusion Type of Study Design}

All journal articles, master's dissertations, and doctoral dissertations reporting on RCTs and published in English/Chinese were included. Conference papers, newspaper articles, nonrandomized clinical studies, case reports, and reviews were excluded. Two-arm trials that only compared different treatment times or frequencies in the same treatment regimen were also excluded.

\section{Type of Participant}

We included trials with all patients who met the NLBP diagnostic criteria, regardless of patient sex, age, ethnic group, disease etiology, and disease severity. Trials with patients diagnosed with LBP not belonging to any nonspecific subtype were excluded.

\section{Type of Intervention}

We limited the intervention types in the included studies for both the experimental and control groups, as follows: (1) intervention group: manual acupuncture, moxibustion, warm acupuncture, auricular needling, electroacupuncture, and fire acupuncture. These six types of acupuncture were used alone or combined with each other or with conventional medicines (eg, NSAIDs, opioid analgesics, and paracetamol). (2) control group: six types of acupuncture method (the same as the intervention group), conventional medicines (eg, NSAIDs, opioid analgesics, and paracetamol), routine care (eg, waiting for treatment, bed rest, and hot packs), placebo (sham acupuncture or other placebo treatments). These nine interventions were used alone or in combination. However, we did not limit treatment factors such as the selection of acupoints, manipulation, treatment time, and number of treatments. In addition, we excluded studies of acupuncture combined with traditional herbal medicine, tuina, transcutaneous electrical nerve stimulation, and acupoint embedding therapy for NLBP. If the intervention method for the different groups in multi-arm studies was identical (but used different intervention measures), we combined the data for the two groups.

\section{Type of Outcome Measure}

We primarily aimed to compare the efficacy and safety of different acupuncture methods for NLBP. The primary outcome was a reduction in visual analog scale (VAS) score after treatment. Secondary outcomes were a reduction in Oswestry Disability Index (ODI) score after treatment and the incidence of adverse events (AE). The reduction in VAS and ODI scores was used to evaluate the pain intensity and functional status of NLBP patients, and the incidence of AEs was used to evaluate the safety of acupuncture methods. RCTs that used numeric rating scales or verbal rating scales were included and the scores converted to VAS scores on a $0-10$ scale for the statistical analysis. The endpoint for outcome evaluation was the first evaluation after the last treatment.

\section{Study Screening Process}

First, all reviewers were professionally trained to conduct the review. To select the studies, the reviewers first read the title/abstract of the article to identify duplicate studies, then they uploaded eligible articles to a database built using NoteExpress V.3.2.0. Further, two authors (LW and ZY) independently screened the articles from several databases back-to-back to ensure reliability. If there was a disagreement between the authors, a third author made the judgment (LZ). Another author (YZ) conducted a manual search of three journals, including Acupuncture Research.

\section{Data Extraction and Analysis}

We extracted the data into Microsoft Excel 2016 and the extraction process was independently completed by two authors (MS and YY). These authors discussed any 
disagreements, and sought the opinion of a third author (LZ) if an agreement could not be reached. The extracted data included title, first author, year of publication, country of study, course of disease, sample size, allocation ratio, intervention measures, duration of treatment, and results.

Data analysis mainly used Review Manager (Revman 5.3) and Aggregate Data Drug Information System (ADDIS Version 1.16.8). Standardized mean differences (SMD) and 95\% confidence intervals (CIs) represented continuous outcomes. Pairwise meta-analysis was used for direct comparisons. A fixed effects model was used for overall analysis in the absence of obvious heterogeneity $\left(\mathrm{I}^{2}<50 \%\right)$; otherwise, a random effects model was used. A Markov chain Monte Carlo method was used to conduct NMA using the ADDIS. Subsequently, we generated various NMA plots in Stata software (Version 16.0 MP). To separate the evidence of each comparison into direct and indirect evidence, the node-splitting method was used. The surface under the cumulative ranking curve (SUCRA) was used to rank acupuncture methods. Finally, a cluster ranking plot was generated to evaluate the comprehensive ability of acupuncture methods to reduce NLBP pain and disability.

\section{Risk of Bias Assessment}

Two authors (MS and YY) independently evaluated the risk of bias in RCTs in accordance with the Cochrane Collaboration risk of bias tool. Seven criteria were applied: sequence generation, allocation concealment, blinding of participants and personnel, blinding of outcome assessment, incomplete outcome data, selective reporting, and other bias. We classified each item as low, high, or unclear risk. In case of doubt, a third author (LZ) made the judgment. We used the following criteria for assessing the overall risk of bias: ${ }^{27}$ if all seven domains of a trial were judged as low risk, or there were less than three unclear risks, the trial was judged as low risk. If a trial had more than two high-risk items, it was considered high risk. Other trials were classified as having unclear risk.

\section{Publication Bias Assessment}

We generated a comparison-adjusted funnel plot to detect reporting bias.

\section{Sensitivity Analysis}

We evaluated the robustness of each result by conducting sensitivity analyses that excluded small sample studies $(<60)$, large-sample studies $(>200)$, or high-risk bias studies.

\section{Patient Involvement}

Patients were not involved in the design, conduct, reporting, or dissemination of this research.

\section{Results}

\section{Literature Search}

We identified 5039 records from the databases and trial registries and selected 150 possible eligible citations for full-text review. After excluding 120 reports, 30 trials $^{5,8,28-55}$ with 3196 participants (all references to included trials are shown in Additional file 4) met the inclusion criteria. Two articles ${ }^{50,53}$ were only used for quality evaluation and were not included in the metaanalysis (one had missing data and the author could not be contacted, and the other used median and quartile data). A flow chart (Figure 1) illustrates the selection process.

A total of 30 RCTs were included in the study. Most trials were carried out in China (20/30); ${ }^{28-42,44-48}$ the others were conducted in Brazil (4/30), ${ }^{49-51,54}$ Iran $(1 / 30),{ }^{5}$ England $(1 / 30),{ }^{8}$ Spain $(1 / 30),{ }^{52}$ Norway $(1 / 30),{ }^{53}$ Korean $(1 / 30)^{55}$ and both China and Iran (1/30). ${ }^{43}$ Of all trials, 4 were on acute NLBP, ${ }^{35,52-54} \quad 19$ on chronic NLBP, ${ }^{5,28-31,33,36-40,42,44,47-51,55}$ and 7 on unclear NLBP. ${ }^{8,32,34,41,43,45,46}$ The included studies had an average sample size of 107 (range 56-275). Only one trial had a 2:1 allocation ratio; ${ }^{8}$ the other trials had a 1:1 ratio. A total of 3167 patients (mean age range: $31.57-60.77$ years) were included in the records (29 patients were lost to follow-up), of who $53.96 \%(n=1709)$ were women. The studies included 12 acupuncture intervention methods in addition to conventional medicines, routine care, and placebo. Details of each trial are shown in the Additional file 1.

Figures 2 and 3 show the results of the Cochrane risk of bias assessment for the 30 trials. Three trials ${ }^{31,38,39}$ were classified as high risk owing to inadequate random sequence generation, such as including different groups based on the treatment approach, baseline balance, and patient wishes. Twelve trials ${ }^{8,29,40,43,46,49-55}$ were classified as high risk because they did not use the correct blinding method. Four trials ${ }^{8,40,43,55}$ were classified as high risk owing to incomplete outcome data because of high patient attrition or inconsistencies in the amount of attrition between groups. Regarding other bias, only one study ${ }^{51}$ was rated as having a high risk of bias owing to a baseline imbalance. Briefly, approximately $16.67 \%$ of the studies ${ }^{8,40,43,51,55}$ were classified as having a high risk of overall bias. 


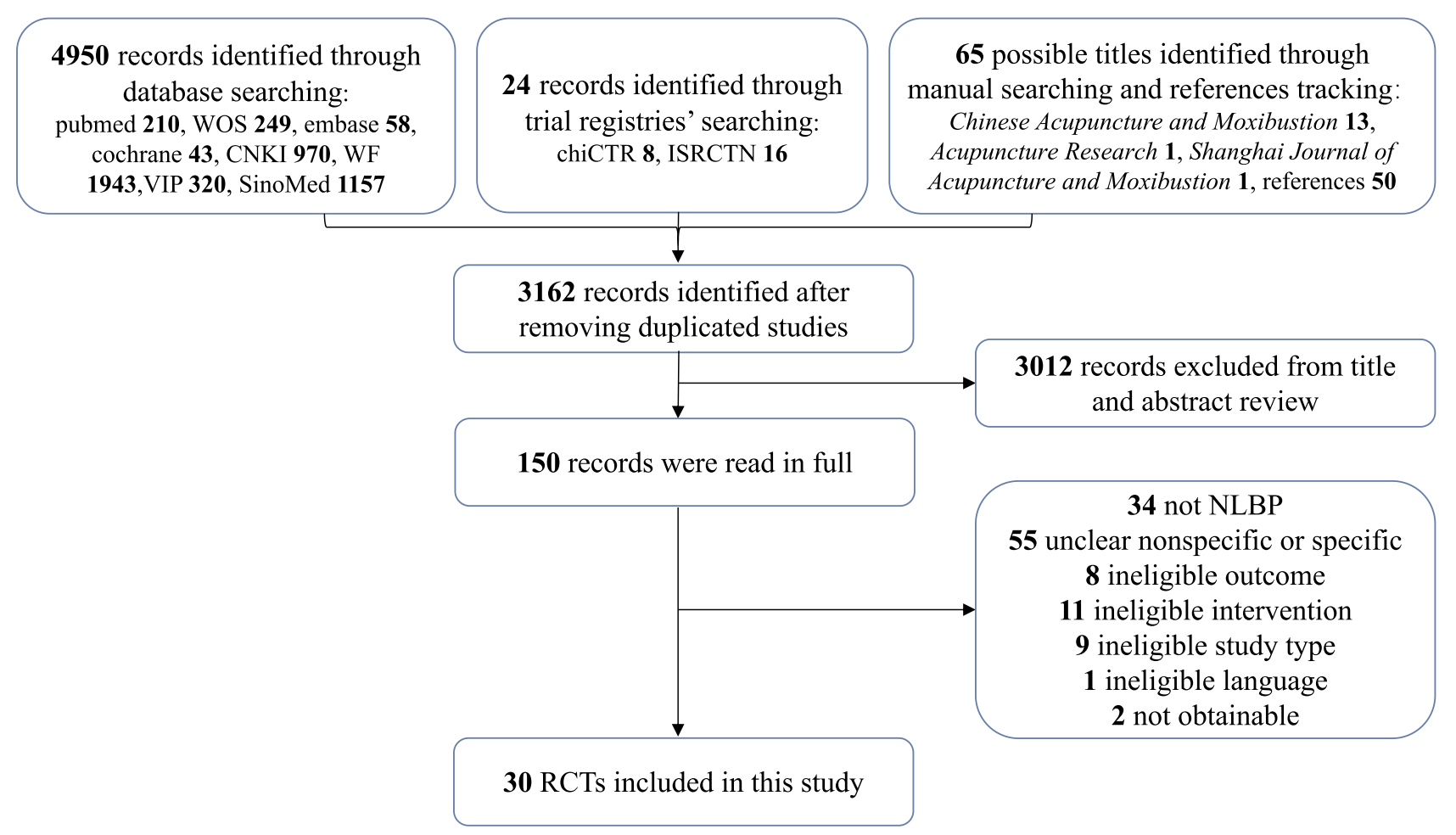

Figure I Flow chart of study selection.

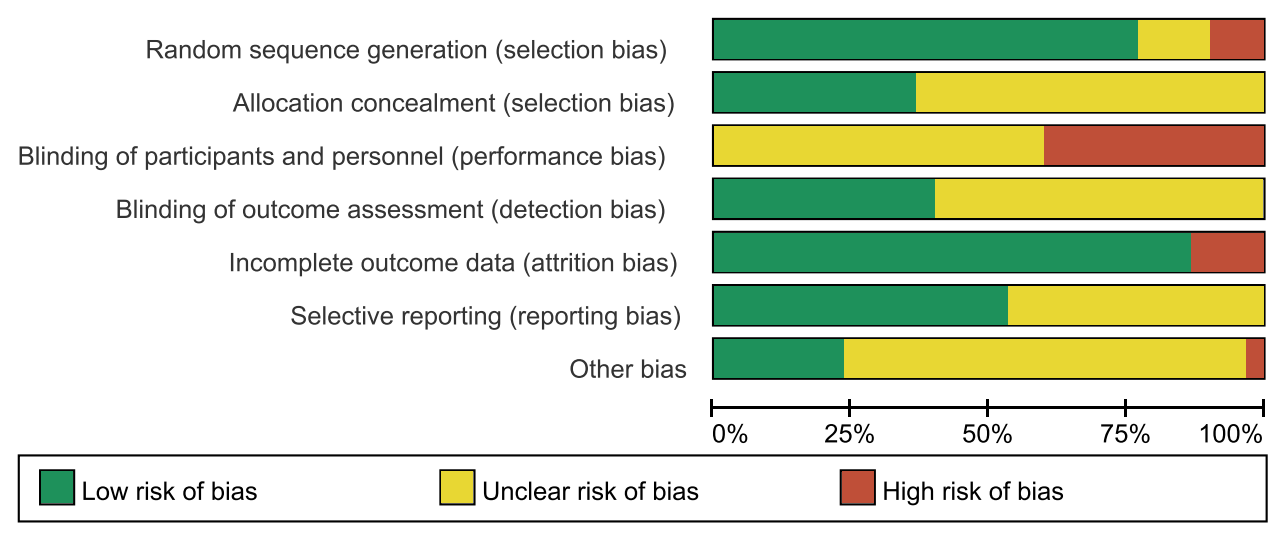

Figure 2 Risk of bias graph.

\section{Pairwise Meta-Analysis}

We performed pairwise meta-analyses to compare the two interventions with a combined effect size. The results are shown in Table 2. Manual acupuncture was more efficacious than conventional medicines ( 2 RCTs; SMD $1.03,95 \%$ CI 0.53 to $\left.1.53 ; \mathrm{I}^{2}=0, p=0.51\right)$ and routine care (3 RCTs; SMD 1.05, 95\% CI 0.67 to 1.43 ; $\left.\mathrm{I}^{2}=0, p=0.39\right)$ in terms of VAS score. However, manual acupuncture had a lower effect than fire acupuncture plus manual acupuncture (2 RCTs; SMD $-2.29,95 \%$ CI -2.64 to $-1.94 ; \mathrm{I}^{2}=0, p=0.92$ ).
Similar to manual acupuncture, moxibustion had a greater effect than conventional medicines (2 RCTs, SMD $0.98,95 \%$ CI 0.37 to $1.60 ; \mathrm{I}^{2}=80, p=0.03$ ). There were no differences in VAS score between manual acupuncture and electroacupuncture, and placebo, and manual acupuncture plus conventional medicines. Comparison of two interventions with a combined effect size showed that routine care had a similar effect on ODI score as manual acupuncture (Table 3). Tables 2 and 3 show additional results of the pairwise metaanalysis and heterogeneity estimates. 


\begin{tabular}{|c|c|c|c|c|c|c|c|}
\hline & 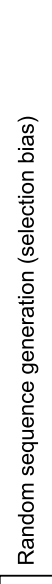 & 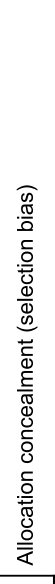 & 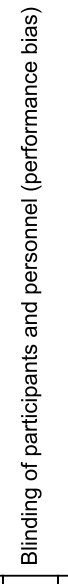 & 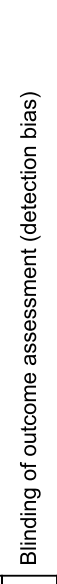 & 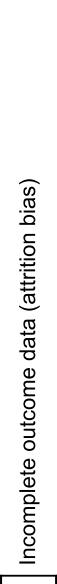 & 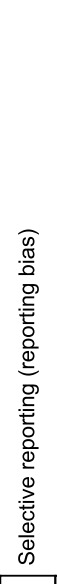 & 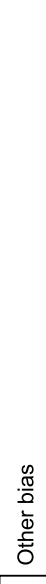 \\
\hline A Ushinohama 2016 [50] & + & + & $\theta$ & + & + & + & $?$ \\
\hline Cheng J 2020 [30] & + & $?$ & $?$ & $?$ & + & $?$ & $?$ \\
\hline Chen P 2015 [38] & $\theta$ & $?$ & $?$ & $?$ & + & $?$ & $?$ \\
\hline $\mathrm{Hu} \times 2020$ [32] & + & + & $?$ & $?$ & + & + & $?$ \\
\hline H Vakilsamad 2018 [43] & + & + & $\theta$ & + & $\theta$ & $?$ & $?$ \\
\hline J Comachio 2020 [49] & + & + & $\theta$ & + & + & + & + \\
\hline $\operatorname{Jin} \times 2018$ [42] & + & + & $?$ & + & + & $?$ & $?$ \\
\hline J Vas 2012 [52] & + & + & $\theta$ & + & + & + & + \\
\hline J Zaringhalam 2010 [5] & + & + & $?$ & $?$ & + & + & + \\
\hline K Thomas 2006 [8] & + & + & $\theta$ & + & $\theta$ & + & + \\
\hline Lai P 2019 [46] & + & $?$ & $\theta$ & $?$ & + & $?$ & $?$ \\
\hline Li J 2017 [47] & + & $?$ & $?$ & $?$ & + & $?$ & $?$ \\
\hline Pan L 2018 [34] & + & $?$ & $?$ & $?$ & + & $?$ & $?$ \\
\hline P Leite 2018 [51] & + & + & 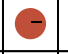 & + & + & + & $\theta$ \\
\hline T Hasegawa 2014 [54] & + & $?$ & $\theta$ & + & + & + & + \\
\hline Tian J 2019 [48] & $?$ & $?$ & $?$ & $?$ & + & + & $?$ \\
\hline T Skonnord 2020 [53] & + & + & $O$ & + & + & + & + \\
\hline Wang X 2018 [39] & $\theta$ & $?$ & $?$ & $?$ & + & $?$ & $?$ \\
\hline Xiao J 2012 [33] & + & $?$ & $?$ & $?$ & + & $?$ & $?$ \\
\hline Yang Y 2017 [45] & + & $?$ & $?$ & + & + & $?$ & $?$ \\
\hline Yang Y 2019 [37] & + & $?$ & $?$ & $?$ & + & $?$ & $?$ \\
\hline Y Cho 2013 [55] & + & $?$ & 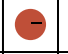 & + & $\theta$ & + & + \\
\hline Ye R 2017 [44] & $?$ & $?$ & $?$ & $?$ & + & + & $?$ \\
\hline Yin X 2019 [31] & $\theta$ & $?$ & $?$ & $?$ & + & + & $?$ \\
\hline Yuan Q 2016 [29] & + & + & $\theta$ & $?$ & + & $?$ & $?$ \\
\hline Yuan S 2016 [35] & $\oplus$ & $?$ & $?$ & + & + & + & $?$ \\
\hline Zhang A 2020 [28] & $?$ & $?$ & $?$ & $?$ & + & $?$ & $?$ \\
\hline Zhang H 2014 [41] & + & $?$ & $?$ & $?$ & + & $?$ & $?$ \\
\hline Zhang W 2018 [40] & + & $?$ & O & $?$ & $\theta$ & + & $?$ \\
\hline Zhou J 2020 [36] & $?$ & $?$ & $?$ & $?$ & + & + & $?$ \\
\hline
\end{tabular}

Figure 3 Risk of bias summary.

\section{Network Meta-Analysis}

Figures 4 and 5 show the NMA network plot for different interventions. A total of 26 trials $^{8,28-49,51,54,55}$ with 2383 participants and 15 interventions reported usable VAS data (Figure 4). Of these, more participants received manual acupuncture than other interventions, and studies comparing manual acupuncture and electroacupuncture, manual acupuncture and placebo were represented most. ODI scores were reported in 12 studies $^{5,28-33,35,40,42,47,48}$ involving 1115 participants and 11 therapies (Figure 5). Similarly, the largest sample size was for the manual acupuncture group, and studies comparing manual acupuncture with routine care were represented most.

Valid NMA results depend on the evidence network being internally consistent: direct and various sources of indirect evidence should be in agreement. ${ }^{56}$ In this study, we used the segmented node method to test for inconsistencies in the NMA (Additional file 2). The results showed no statistical significance for the direct or indirect comparisons of each segmentation node ( $p>0.05$ ), indicating that there was no evidence of design inconsistency. We tested the convergence of the model, and the results were excellent (the potential scale reduction factor value was equal to 1, Additional file 3).

In terms of VAS reduction, Figure 6 shows that all treatment methods were superior to routine care except conventional medicines, placebo, and electroacupuncture plus conventional medicines. We also found that manual acupuncture, moxibustion, auricular needling, fire acupuncture, electroacupuncture plus warm acupuncture, manual acupuncture plus moxibustion, manual acupuncture plus conventional medicines, and fire acupuncture plus manual acupuncture were all better than conventional medicines. Furthermore, fire acupuncture plus manual acupuncture showed better efficacy than manual acupuncture, electroacupuncture, moxibustion, placebo, electroacupuncture plus conventional medicines, and manual acupuncture plus conventional medicines in reducing VAS scores. In addition, electroacupuncture and placebo were less effective than auricular needling, fire acupuncture, electroacupuncture plus warm acupuncture, and fire acupuncture plus manual acupuncture. However, the NMA results showed no significant difference between any two intervention methods in reducing ODI score.

We used a consistent model to perform NMA using ADDIS, and generated a ranking probability matrix (Figures 7 and 8). Figure 7 shows that routine care was the least effective treatment for VAS score reduction. Of the 15 
Table 2 Pairwise Meta-Analysis of Reduction in VAS Score

\begin{tabular}{|c|c|c|c|c|}
\hline Comparison & Number & SMD $(95 \% \mathrm{Cl})$ & $I^{2}(\%)$ & $\mathbf{p}$ \\
\hline$A$ vs $B$ & 4 & $-0.17(-1.25,0.91)$ & 93 & $<0.00001$ \\
\hline A vs $D$ & 2 & $1.03(0.53,1.53)$ & 0 & 0.51 \\
\hline$A$ vs $E$ & 4 & $0.24(-0.42,0.89)$ & 83 & 0.0006 \\
\hline$A$ vs $F$ & 3 & $1.05(0.67,1.43)$ & 0 & 0.39 \\
\hline A vs I & I & $-1.44(-1.86,-1.02)$ & - & - \\
\hline A vs $M$ & I & $-0.67(-1.19,-0.15)$ & - & - \\
\hline A vs $N$ & 2 & $-0.31(-0.68,0.06)$ & 0 & 0.87 \\
\hline A vs $O$ & 2 & $-2.29(-2.64,-1.94)$ & 0 & 0.92 \\
\hline$B$ vs $D$ & 1 & $0.65(0.13,1.17)$ & - & - \\
\hline$B$ vs $E$ & I & $0.63(-0.39,1.64)$ & - & - \\
\hline$B$ vs $G$ & I & $-0.59(-1.21,0.03)$ & - & - \\
\hline B vs $L$ & I & $-1.34(-2.01,-0.66)$ & - & - \\
\hline C vs D & 2 & $0.98(0.37,1.60)$ & 80 & 0.03 \\
\hline$C$ vs $F$ & 1 & $1.39(0.97,1.81)$ & - & - \\
\hline$D$ vs $F$ & I & $0.10(-1.03,1.24)$ & - & - \\
\hline D vs I & I & $-1.30(-1.80,-0.79)$ & - & - \\
\hline D vs J & I & $-1.79(-2.20,-1.38)$ & - & - \\
\hline $\mathrm{D}$ vs $\mathrm{N}$ & 1 & $-1.13(-2.28,0.03)$ & - & - \\
\hline $\mathrm{D}$ vs $\mathrm{O}$ & I & $-1.29(-1.68,-0.89)$ & - & - \\
\hline$E$ vs $F$ & I & $0.70(0.13,1.27)$ & - & - \\
\hline E vs $\mathrm{H}$ & I & $-1.37(-1.88,-0.86)$ & - & - \\
\hline $\mathrm{F}$ vs $\mathrm{K}$ & I & $-1.50(-2.01,-0.99)$ & - & - \\
\hline F vs $N$ & 1 & $-1.18(-2.39,0.04)$ & - & - \\
\hline G vs $L$ & I & $-0.73(-1.36,-0.11)$ & - & - \\
\hline$G$ vs $M$ & I & $-1.32(-1.89,-0.76)$ & - & - \\
\hline
\end{tabular}

Notes: The bold font indicates a statistical difference. $\mathrm{A}=$ manual acupuncture; $\mathrm{B}=$ electroacupuncture; $\mathrm{C}=$ moxibustion; $\mathrm{D}=$ conventional medicines; $\mathrm{E}=$ placebo; $\mathrm{F}=$ routine care; $\mathrm{G}=$ warm acupuncture; $\mathrm{H}=$ auricular needling; $\mathrm{I}=$ fire acupuncture; $\mathrm{J}=$ electroacupuncture plus conventional medicines; $\mathrm{K}=$ electroacupuncture plus moxibustion; $\mathrm{L}=$ electroacupuncture plus warm acupuncture; $\mathrm{M}=$ manual acupuncture plus moxibustion; $\mathrm{N}=$ manual acupuncture plus conventional medicines; $\mathrm{O}=$ fire acupuncture plus manual acupuncture.

Table 3 Pairwise Meta-Analysis for Reduction in ODI Score

\begin{tabular}{|c|c|c|c|c|}
\hline Comparison & Number & SMD ( $95 \% \mathrm{Cl})$ & $I^{2}(\%)$ & $\mathbf{p}$ \\
\hline$A$ vs $B$ & 1 & $0.57(0.06,1.09)$ & I & I \\
\hline$A$ vs $D$ & 1 & $0.06(-0.47,0.58)$ & 1 & 1 \\
\hline$A$ vs $E$ & 1 & $0.40(-0.16,0.96)$ & l & 1 \\
\hline$A$ vs $F$ & 3 & $0.32(-0.13,0.77)$ & 68 & 0.04 \\
\hline A vs $M$ & I & $-0.68(-1.20,-0.16)$ & I & 1 \\
\hline A vs $N$ & I & $-0.63(-1.03,-0.23)$ & l & I \\
\hline B vs $D$ & I & $3.46(2.65,4.28)$ & l & I \\
\hline C vs $\mathrm{F}$ & 1 & $0.87(0.48,1.27)$ & I & 1 \\
\hline D vs I & I & $-0.88(-1.36,-0.4 I)$ & I & 1 \\
\hline E vs $F$ & I & $0.47(-0.09,1.04)$ & I & 1 \\
\hline F vs $\mathrm{K}$ & I & $-1.03(-1.5 I,-0.55)$ & l & I \\
\hline G vs $M$ & I & $-1.56(-2.14,-0.98)$ & I & 1 \\
\hline
\end{tabular}

Notes: The bold font indicates a statistical difference. $A=$ manual acupuncture; $B=$ electroacupuncture; $\mathrm{C}=$ moxibustion; $\mathrm{D}=$ conventional medicines; $\mathrm{E}=$ placebo; $\mathrm{F}=$ routine care; $G$ = warm acupuncture; $I$ = fire acupuncture; $K$ = electroacupuncture plus moxibustion; $\mathrm{M}=$ manual acupuncture plus moxibustion; $\mathrm{N}=$ manual acupuncture plus conventional medicines. treatments, fire acupuncture plus manual acupuncture, auricular needling, and electroacupuncture plus warm acupuncture ranked high in reducing VAS score. Figure 8 shows that of the 11 treatment methods, manual acupuncture plus conventional medicines, moxibustion, and manual acupuncture plus moxibustion were the three best treatment methods to reduce ODI score. For the SUCRA scores, see Additional file 5.

\section{Sensitivity Analysis}

Sensitivity analyses were first conducted for all pairwise meta-analyses after excluding trials with a high risk of bias. There was little or no change to the results. After removing small sample studies $(n<60)$, the results showed that manual acupuncture had the same effect in reducing VAS score as conventional medicines (SMD 0.70, 95\% CI 


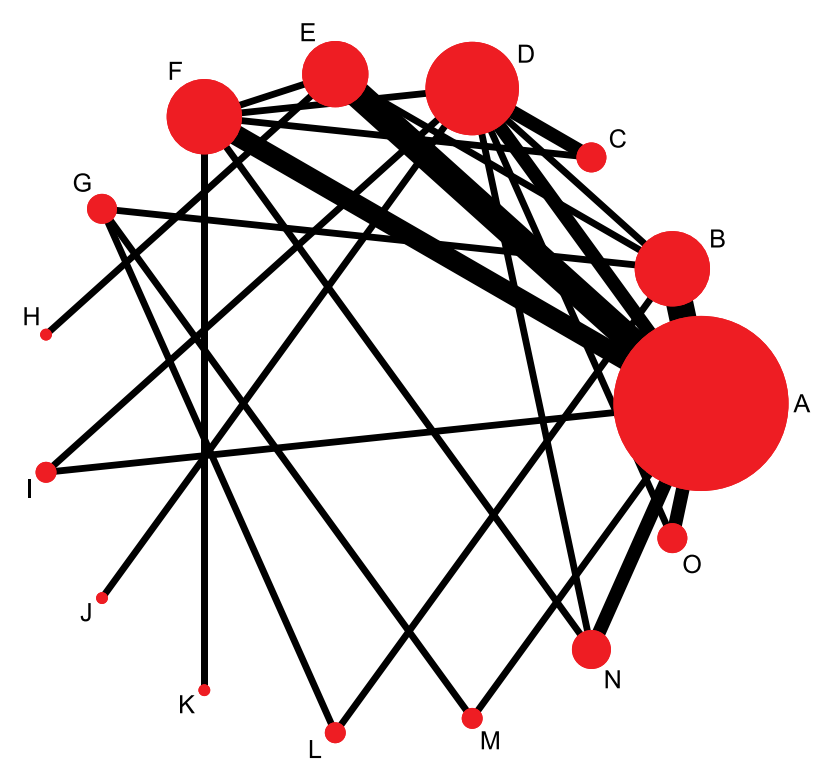

Figure 4 Network plot of VAS scores.

Notes: $\mathrm{A}$ = manual acupuncture; $\mathrm{B}=$ electroacupuncture; $\mathrm{C}=$ moxibustion; $\mathrm{D}=$ conventional medicines; $\mathrm{E}=$ placebo; $\mathrm{F}=$ routine care; $\mathrm{G}=$ warm acupuncture; $\mathrm{H}=$ auricular needling; I= fire acupuncture; $J=$ electroacupuncture plus conventional medicines; $K=$ electroacupuncture plus moxibustion; $L=$ electroacupuncture plus warm acupuncture; $\mathrm{M}=$ manual acupuncture plus moxibustion; $\mathrm{N}=$ manual acupuncture plus conventional medicines; $\mathrm{O}=$ fire acupuncture plus manual acupuncture.

Figure 5 Network plot of ODI scores.

Notes: $A=$ manual acupuncture; $B=$ electroacupuncture; $C=$ moxibustion; $D=$ conventional medicines; $E=$ placebo; $F=$ routine care; $G=$ warm acupuncture; $I=$ fire acupuncture; $\mathrm{K}=$ electroacupuncture plus moxibustion; $\mathrm{M}=$ manual acupuncture plus moxibustion; $\mathrm{N}=$ manual acupuncture plus conventional medicines. 


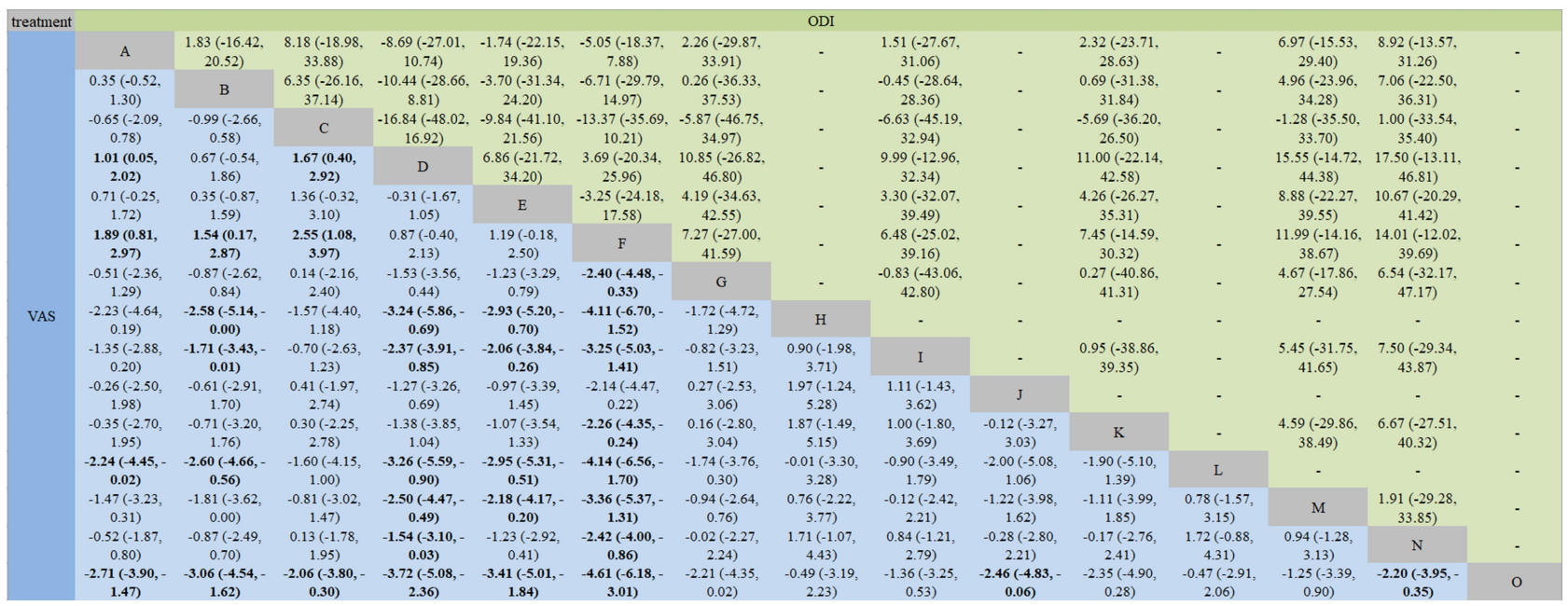

Figure 6 Network meta-analysis results for VAS and ODI scores.

Notes: The bold font indicates a statistical difference. $A=$ manual acupuncture; $B=$ electroacupuncture; $C=$ moxibustion; $D=$ conventional medicines; $E=$ placebo; $F=$ routine care; $\mathrm{G}=$ warm acupuncture; $\mathrm{H}=$ auricular needling; I= fire acupuncture; J= electroacupuncture plus conventional medicines; $\mathrm{K}=$ electroacupuncture plus moxibustion; $\mathrm{L}=$ electroacupuncture plus warm acupuncture; $\mathrm{M}=$ manual acupuncture plus moxibustion; $\mathrm{N}=$ manual acupuncture plus conventional medicines; $\mathrm{O}=$ fire acupuncture plus manual acupuncture.

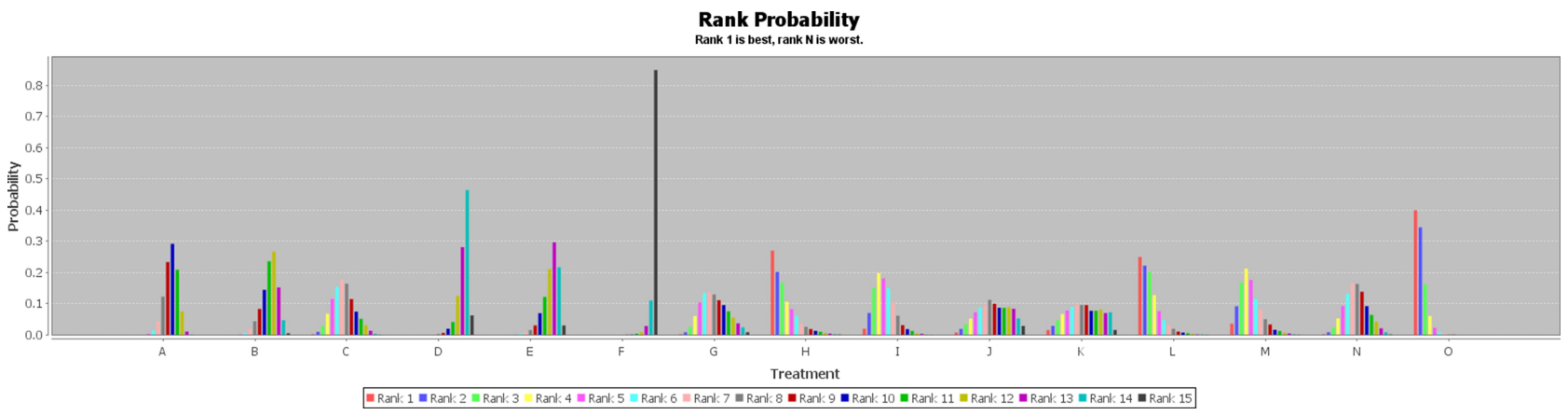

Figure 7 Ranking probability figure for reduction in VAS score.

Notes: $A=$ manual acupuncture; $B=$ electroacupuncture; $C=$ moxibustion; $D=$ conventional medicines; $E=$ placebo; $F=$ routine care; $G=$ warm acupuncture; $H=$ auricular needling; I= fire acupuncture; $\mathrm{J}=$ electroacupuncture plus conventional medicines; $\mathrm{K}=$ electroacupuncture plus moxibustion; $\mathrm{L}=$ electroacupuncture plus warm acupuncture; $\mathrm{M}=$ manual acupuncture plus moxibustion; $\mathrm{N}=$ manual acupuncture plus conventional medicines; $\mathrm{O}=$ fire acupuncture plus manual acupuncture.

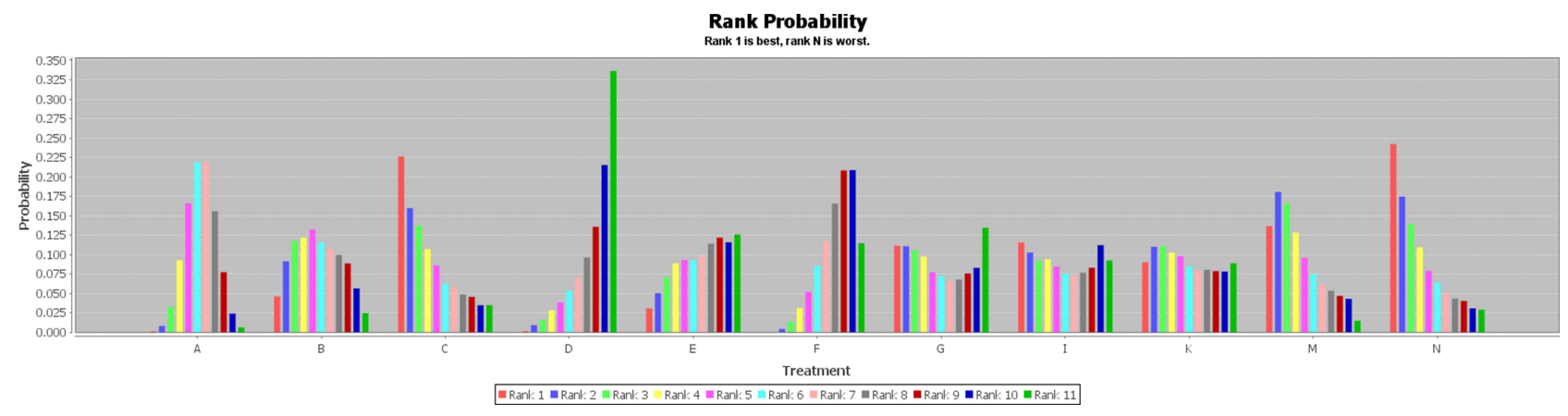

Figure 8 Ranking probability figure for reduction in ODI score.

Notes: $A=$ manual acupuncture; $B=$ electroacupuncture; $C=$ moxibustion; $D=$ conventional medicines; $E=$ placebo; $F=$ routine care; $G=$ warm acupuncture; $I=$ fire acupuncture; $\mathrm{K}=$ electroacupuncture plus moxibustion; $\mathrm{M}=$ manual acupuncture plus moxibustion; $\mathrm{N}=$ manual acupuncture plus conventional medicines. 
-0.39 to 1.79$)$. Finally, large-sample studies $(n>200)$ were excluded, but the meta-analysis results remained unchanged.

\section{Cluster Ranking Results for VAS and ODI Scores}

We conducted a cluster sequencing for interventions that evaluated both VAS and ODI scores (Figure 9), and grouped the treatment approaches into four groups. It was clear that the efficacy of routine care and conventional medicines was much lower than other interventions. Manual acupuncture plus conventional medicines and moxibustion were the two interventions that produced the greatest improvement in ODI score. Additionally, fire acupuncture had an obvious effect in reducing VAS score. The final results showed that manual acupuncture plus moxibustion can achieve good results in improving both VAS and ODI scores.

\section{Adverse Events}

We identified 13 trials $29,30,33-36,38,40,42,45,52,54,55$ that used AEs as outcomes; the results are shown in Figure 10. We approximately divided AEs into seven categories: increased pain, local acupuncture point pain, subcutaneous hematomas, vertigo or headache, stomachache and nausea, minor burns (ie, the burn area is small and involves the superficial epidermis or the superficial dermis), and other symptoms. Manual acupuncture was associated with the greatest number of AE categories (six of the above categories). However, the AE categories with the highest incidences were increased pain, other symptoms, stomachache and nausea $(29.01 \%, 21.37 \%$ and $17.56 \%$, respectively). Routine care was the intervention with the highest incidence of AEs (36.67\%). Briefly, the overall AE rate was $7.70 \%$ for the eight acupuncture methods and $12.24 \%$ for the three other treatments (conventional medicines, routine care, and placebo). No serious AEs occurred in any of the trials.

\section{Publication Bias}

We evaluated the publication bias by examining the adjusted funnel plot (Figures 11 and 12). The results showed that most of the points were evenly distributed on both sides of the midline and concentrated in the middle area. It is reasonable to assume that most of the included studies had moderate sample sizes, and we believe that these studies had a low degree of bias. However, there were a few points outside the two dashed

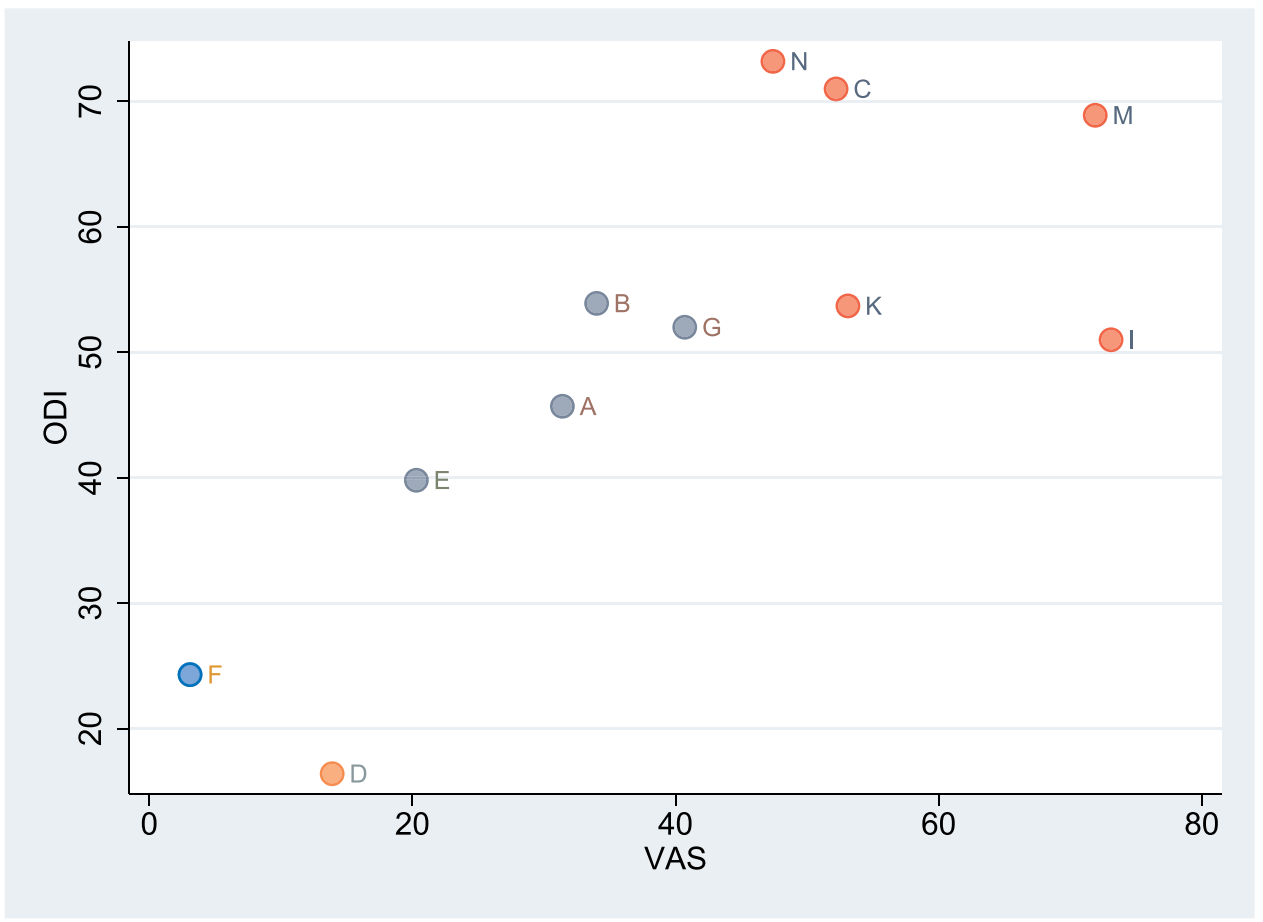

Figure 9 Cluster ranking plot based on reduction in VAS and ODI scores.

Notes: Each color represents a group of interventions that belong to the same cluster. Interventions lying in the upper right corner were more effective than the other interventions. $A=$ manual acupuncture; $B=$ electroacupuncture; $C=$ moxibustion; $D=$ conventional medicines; $E=$ placebo; $F=$ routine care; $G=$ warm acupuncture; $I=$ fire acupuncture; $\mathrm{K}=$ electroacupuncture plus moxibustion; $\mathrm{M}=$ manual acupuncture plus moxibustion; $\mathrm{N}=$ manual acupuncture plus conventional medicines. 


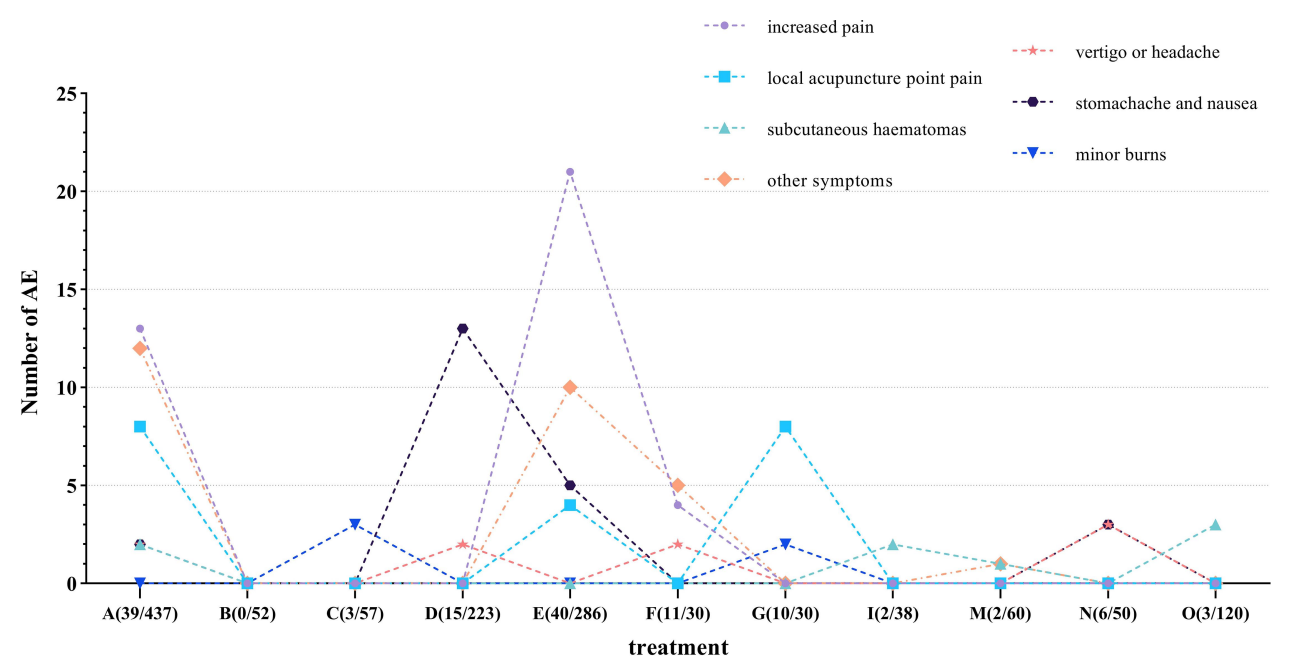

Figure 10 Number of AEs for different treatment measures.

Notes: The data in brackets are the ratio of the number of $A E s$ to the sample size. $A=$ manual acupuncture; $B=$ electroacupuncture; $C=$ moxibustion; $D=$ conventional medicines; $\mathrm{E}=$ placebo; $\mathrm{F}=$ routine care; $\mathrm{G}=$ warm acupuncture; $\mathrm{I}=$ fire acupuncture; $\mathrm{M}=$ manual acupuncture plus moxibustion; $\mathrm{N}=$ manual acupuncture plus conventional medicines; $\mathrm{O}=$ fire acupuncture plus manual acupuncture.

lines, which suggests potential heterogeneity between these studies.

\section{Discussion}

Although there is some evidence that acupuncture is effective in treating $\mathrm{NLBP}^{23,24}$ acupuncture methods are diverse. Acupuncture methods with low curative effect not only affect the condition of patients with NLBP, but also waste medical resources. We therefore attempted to identify optimal acupuncture methods for NLBP treatment. Our quality evaluation of 30 RCTs of acupuncture methods for NLBP identified a few RCTs as high risk of bias studies (5 out of 30 RCTs). The aspect associated with the highest risk of bias was blinding of participants and personnel, which we consider an effect of the

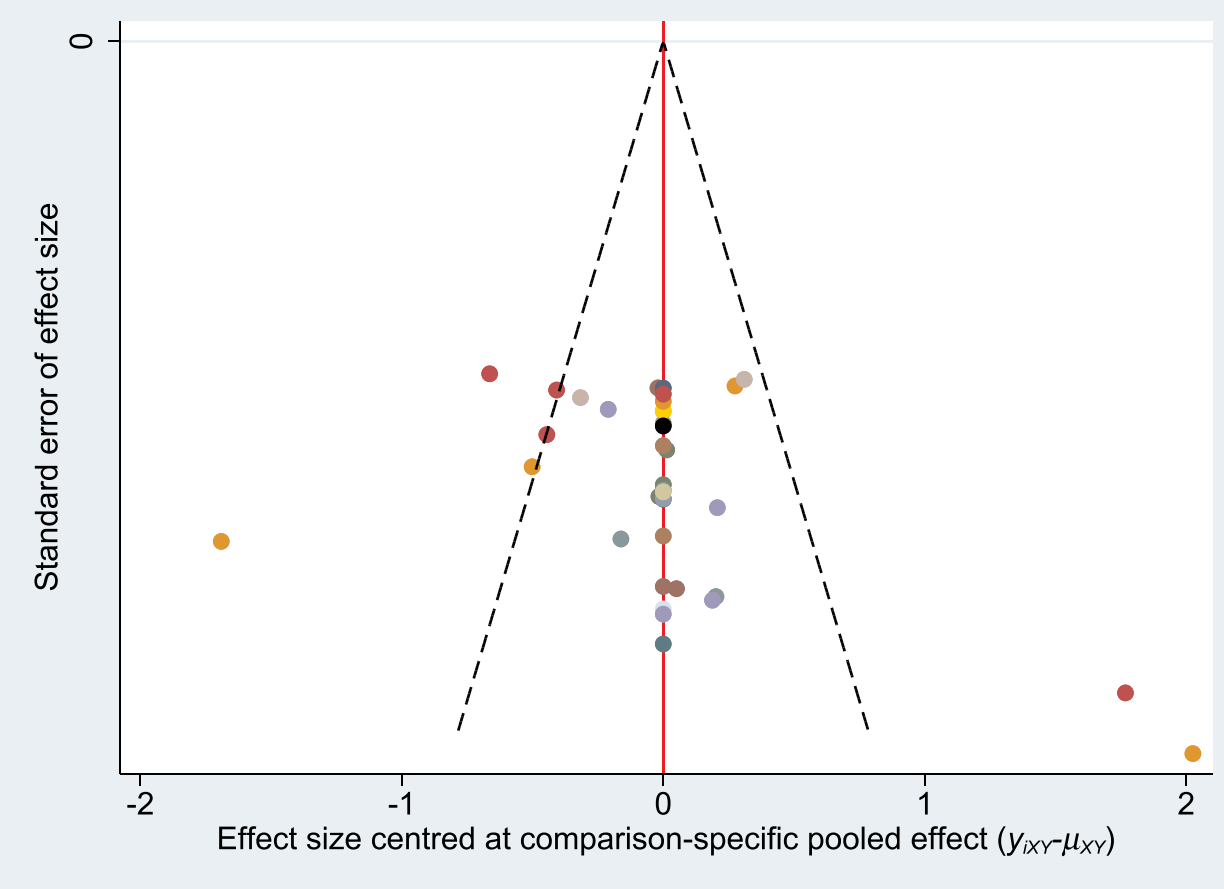

Figure I I Funnel plot for the network meta-analysis of reduction in VAS score. 


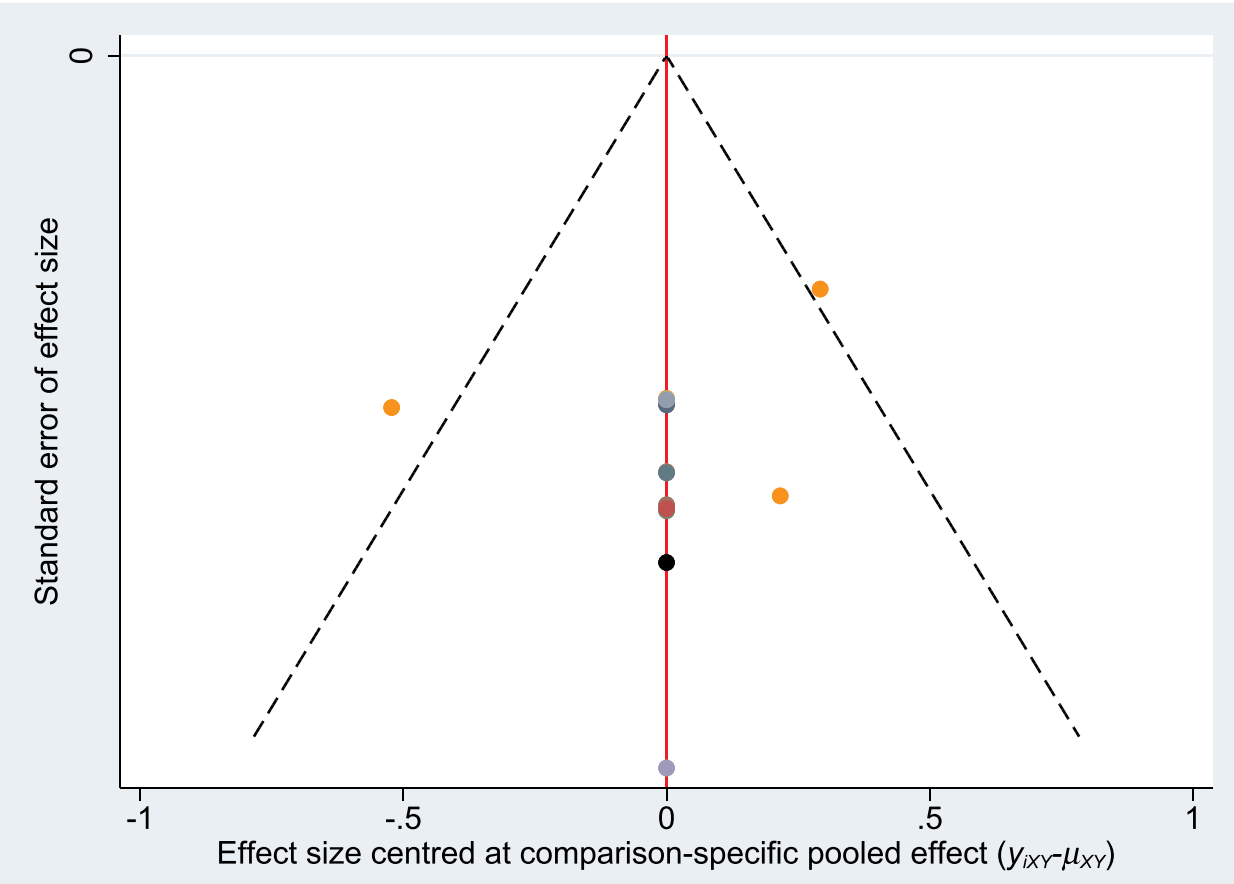

Figure 12 Funnel plot for the network meta-analysis of reduction in ODI score.

unique nature of acupuncture treatment. Unlike traditional drug trials, researchers and acupuncturists cannot be blinded in acupuncture trials. Sensitivity analyses were carefully performed because of the inclusion of some studies with a high risk of bias. The results suggest that most of the results obtained are robust, which strengthens our interpretation of the findings.

We further performed a pairwise meta-analysis and an NMA of 28 RCTs involving 2920 NLBP patients. First, to differentiate between placebo, conventional medicines, and acupuncture methods, we classified bed rest, waiting for treatment, and simple fomentation as routine care. In contrast to some research findings, ${ }^{57,58}$ routine care appeared to have limited efficacy. The pairwise metaanalysis and NMA results indicated that this treatment performed worst in reducing VAS score. Similarly, conventional medicines showed poor efficacy in reducing pain intensity in NLBP patients, a finding supported by the 2016 NICE Draft Guideline. ${ }^{59}$ The guideline points out that the effects of opioids on pain and function in NLBP patients are too limited to be clinically meaningful. In contrast, the pairwise meta-analysis and NMA results showed that fire acupuncture plus manual acupuncture can reduce patient pain. Some studies ${ }^{60,61}$ have shown that, owing to its extremely high temperature, fire acupuncture can rapidly absorb inflammatory factors in the diseased area through the surrounding lymphoid tissue and can inhibit the central nervous system, which may be why it reduces VAS scores in NLBP patients. Additionally, the ranking probability results showed that fire acupuncture plus manual acupuncture was the treatment most likely to reduce VAS score. However, only three trials ${ }^{36,38,39}$ used fire acupuncture plus manual acupuncture, and two of them ${ }^{38,39}$ were classified as high risk of bias trials owing to the the randomization method. Therefore, this finding must be interpreted with caution. We hope that more highquality RCTs are performed to confirm this result.

The NMA results showed no difference in ODI score reduction between any of the 11 treatments when compared with another treatment. Despite this, our ranking probability results suggested that manual acupuncture plus conventional medicines is the optimal treatment for ODI score reduction, followed by moxibustion. The pairwise meta-analysis showed that manual acupuncture plus conventional medicines reduced ODI score compared with manual acupuncture. We also found that moxibustion had a better effect than routine care in reducing ODI score. Therefore, manual acupuncture plus conventional medicines and moxibustion may be useful clinical interventions to reduce body dysfunction in NLBP patients.

The color of the dots in the cluster ranking plot show four intervention categories. Routine care and conventional medicines are in the lower left corner of the plot, 
indicating that these two treatment categories may not the best first treatment options for clinicians. The categories of manual acupuncture, electroacupuncture, placebo, and warm acupuncture are located near the middle of the plot. However, the effects of these four acupuncture methods on VAS and ODI scores was relatively limited, and may not indicate satisfactory clinical effects. Good results in reducing VAS and ODI scores were found for the last set of intervention categories: manual acupuncture plus moxibustion, manual acupuncture plus conventional medicines, moxibustion, fire acupuncture, and electroacupuncture plus moxibustion. Therefore, we recommend that clinical acupuncturists consider using one of these five methods as the first treatment option for NLBP patients with severe pain and disability problems. Overall, manual acupuncture plus moxibustion seems to have the best efficacy in reducing VAS and ODI scores in NLBP patients.

Additionally, we analyzed 12 trials that reported AEs. Increased pain, other symptoms, stomachache and nausea were the most common AEs. Increased pain was caused mainly by placebo, whereas stomachache and nausea were caused by conventional medicines. Therefore, we do not recommend treatment with conventional medicines for NLBP patients with gastrointestinal disease. Moreover, the $\mathrm{AE}$ rate was relatively high for routine care and warm acupuncture. However, it is difficult to draw firm conclusions regarding AEs owing to the small samples in trials of these two intervention types. In summary, the incidence of AEs with acupuncture methods $(7.70 \%)$ was lower than that with other interventions (12.24\%). This indicates that acupuncture treatment methods are safe.

\section{Strengths}

First, this review was conducted according to the PRISMA-NMA $^{26}$ and PRISMA guidelines and checklist. ${ }^{62}$ Additionally, to ensure access to sufficient literature, we searched eight major Chinese and English databases and two clinical trial registries, in addition to manual searching. Second, as RCT is the "gold standard" of clinical trials, we did not include cohort studies or case control studies, which might have affected our final conclusions. Third, we used Bayesian analysis to obtain more accurate estimates than the frequency based approach, and the ADDIS software we used is recommended by some statisticians. ${ }^{63}$ Fourth, our study provides a comprehensive comparison of the efficacy and safety of different acupuncture methods for NLBP and as many as 12 acupuncture methods were examined (not including routine care, conventional medicines, and placebo). Finally, we used cluster ranking to summarize several acupuncture methods that can reduce both VAS and ODI, which may help clinicians to make treatment decisions.

\section{Limitations}

There were several study limitations. First, we collected data for the baseline and the first outcome after the completion of treatment. However, treatment duration and cycle were not consistent across the different studies, which may have increased the risk of study bias. In addition, article language limitations, patient gender, choice of acupuncture points, treatment frequency, and other factors may have caused heterogeneity, but we did not fully examine these factors. Second, the included trials were rigorously evaluated using the Cochrane Collaboration risk of bias tool, but the trial quality was not very high. This may be because we rated single blindness as high risk. Owing to its unique requirements, acupuncture research often involves single-blind designs, which may have increased the risk of bias in the study findings. Third, we detected heterogeneity in the pairwise meta-analysis. Although we adjusted the random effects model, the results may be inaccurate. Fourth, one study ${ }^{5}$ was a fourarm test, and although we broke it down into six different comparisons, each group had a relatively low sample size. Therefore, the findings of this analysis may be unreliable.

\section{Conclusions}

This study provides substantial evidence that acupuncture is more effective in treating NLBP than other interventions (conventional medicines, routine care, and placebo). Manual acupuncture plus moxibustion is the best acupuncture method to reduce pain and disability index scores in NLBP patients. However, stronger head-to-head comparative evidence is needed to confirm this conclusion. We recommend that more high-quality, large-sample, multicenter RCTs are conducted to validate these findings.

\section{Abbreviations}

ADDIS, Aggregate Data Drug Information System; AE, Adverse events; ChiCTR, Chinese Clinical Trial Registry; CIs, Confidence intervals; CNKI, China National Knowledge Infrastructure; ISRCTN, International Standard Randomised Controlled Trial Number Register; LBP, Low back pain; NLBP, Nonspecific low back pain; NMA, Network meta-analysis; NSAIDs, Nonsteroidal 
anti-inflammatory preparations; ODI, Oswestry Disability Index; RCT, Randomized controlled trial; Revman, Review Manager software; SinoMed, Chinese biomedical literature service system; SMD, Standardized mean difference; SUCRA, Surface under the cumulative ranking curve; VAS, Visual analog scale; VIP, VIP Database for Chinese Technical Periodicals; WF, WANFANG Database; WOS, Web of Science.

\section{Data Sharing Statement}

All data relevant to the study are included in the article or uploaded as supplementary information.

\section{Acknowledgment}

Linjia Wang and Zihan Yin contributed equally to this work.

\section{Author Contributions}

LW and ZY designed and registered the project. LW, ZY, and YZ screened the literature. MS and YY extracted the relevant data and evaluated the quality of RCTs. LW and ZY conducted the statistical analysis of the data. LW and $\mathrm{YZ}$ drafted the manuscript. YL and LZ supervised the implementation of the entire study and provided guidance. All authors made a significant contribution to the work reported, whether that is in the conception, study design, execution, acquisition of data, analysis and interpretation, or in all these areas; took part in drafting, revising or critically reviewing the article; gave final approval of the version to be published; have agreed on the journal to which the article has been submitted; and agree to be accountable for all aspects of the work.

\section{Funding}

This project was financially supported by the General Program of the National Natural Science Foundation of China (No. 81973962), the National Key Research and Development Program of China (No. 2019YFC1709701) and the Key Research and Development Program of Sichuan Science and Technology (No. 2019YFS0011).

\section{Disclosure}

The authors declare that they have no competing interests in this work.

\section{References}

1. Elaine A, Mannion AF, Pellisé F, Cedraschi C. Non-specific low back pain. Lancet. 2012;379(9814):482-491. doi:10.1016/S0140-6736(11) 60610-7
2. Lurie JD. What diagnostic tests are useful for low back pain? Best Pract Res Clin Rheumatol. 2005;19:557-575. doi:10.1016/j. berh.2005.03.004

3. Koes BW, van Tulder MW, Thomas S. Diagnosis and treatment of low back pain. BMJ. 2006;332:1430-1434. doi:10.1136/ bmj.332.7555.1430

4. Maher C, Underwood M, Buchbinder R. Non-specific low back pain. Lancet. 2017;389:10070. doi:10.1016/S0140-6736(16)30970-9

5. Zaringhalam J, Manaheji H, Rastqar A, et al. Reduction of chronic non-specific low back pain: a randomized controlled clinical trial on acupuncture and baclofen. Chin Med. 2010;5(1):15. doi:10.1186/ 1749-8546-5-15

6. Nijs J, Apeldoorn A, Hallegraeff H, et al. Low back pain: guidelines for the clinical classification of predominant neuropathic, nociceptive, or central sensitization pain. Pain Physician. 2015;18(3):E333E346. doi:10.36076/ppj.2015/18/E333

7. Tegner H, Frederiksen P, Esbensen BA, et al. Neurophysiological pain education for patients with chronic low back pain: a systematic review and meta-analysis. Clin J Pain. 2018;1.

8. Thomas KJ, MacPherson H, Thorpe L. Randomized controlled trial of a short course of traditional acupuncture compared with usual care for persistent non-specific low back pain. BMJ. 2006;333(7569):623. doi:10.1136/bmj.38878.907361.7C

9. Lee JH, Choi TY, Lee MS, et al. Acupuncture for acute low back pain: a systematic review. Clin J Pain. 2013;29(2):172-185. doi:10.1097/AJP.0b013e31824909f9

10. Deyo RA, Weinstein JN. Low back pain. Adv Pain Res Ther. 1991;18 (2):48.

11. Hoy D, March L, Brooks P, et al. The global burden of low back pain: estimates from the Global Burden of Disease 2010 study. Ann Rheum Dis. 2014;73:968-974. doi:10.1136/annrheumdis-2013-204428

12. Global Burden of Disease Study 2013 Collaborators. Global, regional, and national incidence, prevalence, and years lived with disability for 301 acute and chronic diseases and injuries in 188 countries, 1990-2013: a systematic analysis for the Global Burden of Disease Study 2013. Lancet. 2015;386:743-800.

13. Koes BW, van Tulder M, Lin CW, et al. An updated overview of clinical guidelines for the management of non-specific low back pain in primary care. Eur Spine J. 2010;19(12):2075-2094. doi:10.1007/ s00586-010-1502-y

14. Chou R, Huffman LH. Medications for acute and chronic low back pain: a review of the evidence for an American Pain Society/ American College of Physicians clinical practice guideline. Ann Intern Med. 2007;147(7):505-514. doi:10.7326/0003-4819-1477-200710020-00008

15. Eisenberg DM, Davis RB, Ettner SL, et al. Trends in alternative medicine use in the United States, 1990-1997: results of a followup national survey. JAMA. 1998;280(18):1569-1575. doi:10.1001/ jama.280.18.1569

16. Machado GC, Maher CG, Ferreira PH, et al. Efficacy and safety of paracetamol for spinal pain and osteoarthritis: systematic review and meta-analysis of randomized placebo controlled trials. $B M J$. 2015;350:h1225. doi:10.1136/bmj.h1225

17. Whittle SL, Richards BL, Husni E, et al. Opioid therapy for treating rheumatoid arthritis pain. Cochrane Database Syst Rev. 2011;2011; (11):CD003113.

18. Buch MH, Emery P. New therapies in the management of rheumatoid arthritis. Curr Opin Rheumatol. 2011;23(3):245-251. doi:10.1097/ BOR.0b013e3283454124

19. Solomon DH, Rassen JA, Glynn RJ, et al. The comparative safety of opioids for nonmalignant pain in older adults. Arch Intern Med. 2010;170:1979-1986. doi:10.1001/archinternmed.2010.450

20. Chou R, Turner JA, Devine EB, et al. The eff ectiveness and risks of long-term opioid therapy for chronic pain: a systematic review for a National Institutes of Health Pathways to Prevention Workshop. Ann Intern Med. 2015;162:276-286. doi:10.7326/M14-2559 
21. Dowell D, Haegerich TM, Chou R. CDC guideline for prescribing opioids for chronic pain -United States, 2016. JAMA. 2016;315:1624-1645. doi:10.1001/jama.2016.1464

22. Lee J, Gupta S, Price C, et al. Low back and radicular pain: a pathway for care developed by the British Pain Society. $\mathrm{Br}$ $J$ Anaesth. 2013;2013;(1):112-120.

23. Furlan AD, Tulder MWV, Cherkin D, et al. Acupuncture and dryneedling for low back pain. Cochrane Database Syst Rev. 2005;1(1): CD001351.

24. Liu L, Skinner M, McDonough S, et al. Acupuncture for low back pain: an overview of systematic reviews. Evid Based Complement Alternat Med. 2015;2015:328196.

25. Mu J, Furlan AD, Lam WY, et al. Acupuncture for chronic nonspecific low back pain. Cochrane Database Syst Rev. 2020;(12): CD013814.

26. Hutton B, Salanti G, Caldwell DM, et al. The PRISMA extension statement for reporting of systematic reviews incorporating network meta-analyses of health care interventions: checklist and explanations. Ann Intern Med. 2015;162(11):777-784. doi:10.7326/M14-2385

27. Ye L, Jiaqi Z, Jianchao W, et al. Comparative efficacy and safety of anti-vascular endothelial growth factor regimens for neovascular age-related macular degeneration: systematic review and Bayesian network meta-analysis. Ther Adv Chronic Dis. 2020;11:2040622320953349. doi:10.1177/2040622320953349

28. Zhang AF. The therapeutic value of acupuncture prescription in chronic nonspecific low back pain. Heilongjiang $J$ Trad Chin Med. 2020;309(01):314-315.

29. Yuan QL, Liu L, Ma JT, et al. A clinical study of acupuncture therapy for treatment of chronic nonspecific low back pain. J Trad Chin Orthoped Traumatol. 2016;2016;(6):12-17.

30. Cheng J. Clinical Observation on Acupuncture and Du-Moxibustion in Treating Chronic Non-Specific Low Back Pain (Cold-Dampness Type) [Master]. Hubei University of Traditional Chinese Medicine; 2020.

31. Yin XZ, Xu FL, Xie SJ. Clinical observation of acupuncture intervention on chronic nonspecific low back pain. J Commun Med. 2019;17(23):1503-1506.

32. Hu XW, Tang LM, Deng CY, et al. Effect of long-snake moxibustion on nonspecific low back pain with symptom of cold and dampness. Chin Acupuncture Moxibustion. 2020;40(07):713-716.

33. Xiao J, Jin T, Chen JF. Clinical observation of silver needle combined with paracetamol oxycodone in the treatment of chronic low back pain. Chin J Integr Trad West Med. 2012;32(10):1430-1431.

34. Pan L. Clinical Observation of Moxibustion Treatment of Nonspecific Low Back Pain[Master]. Zhejiang University of Traditional Chinese Medicine; 2018.

35. Yuan SG, Xu MK, Zhou L, et al. Comparison of rehabilitation between traditional acupuncture and bed rest intervention for acute nonspecific low back pain. Hainan Med J. 2016;27(02):306-307.

36. Zhou J, Fan Q, He Z. Observation on clinical effect of fire-needling as main therapy for chronic nonspecific low back pain. $J$ Guangxi Med Univ. 2020;37(07):1326-1330.

37. Yang Y, Zhao J, Zhou XP, et al. Clinical controlled trial of chronic nonspecific low back pain with he's fire needle therapy. J Changchun Univ Chin Med. 2019;35(01):88-90.

38. Chen P, Guo J, Liu HL, et al. Clinical observation of 120 cases of chronic nonspecific low back pain treated with Heshi fire needle therapy and millineedle therapy. $J$ Trad Chin Med. 2015;56 (10):851-854.

39. Wang XW. Effect of HE's fire needle acupuncture in the treatment of chronic nonspecific lumbar pain. Jilin J Chin Med. 2018;38 (09):1098-1100.

40. Zhang WY, Ding Y. Effect of filiform fire-needling therapy on the improvements of clinical symptoms and therapeutic satisfaction in NLBP patients through pricking starting and terminal points. $J$ Clin Acupuncture Moxibustion. 2018;34(08):47-51.
41. Zhang HY. Clinical observation of ginger moxibustion in the treatment of nonspecific low back pain. J Sichuan Trad Chin Med. 2014;32(06):159-160.

42. Jin XF, Li R, Chen LF, et al. A randomized controlled pilot on chronic non-specific low back pain treated with the superficial needling therapy combined with mild moxibustion. Chin Acupuncture Moxibustion. 2018;38(10):1058-1062.

43. Hossein V. Clinical Research on Low Back Pain Treatment by Auricular Acupressure with Magnetic Pellet[Master]. Beijing University of Traditional Chinese Medicine; 2018.

44. Ye R, Zhang GC, Zhao J, et al. Clinical study of electro-acupuncture in treatment of chronic nonspecific low back pain. Acta Chin Med. 2017;32(09):1796-1798.

45. Yang YQ, Zhang ZL, Li S, et al. Effect observation of electro acupuncture at Ashiacupoints for treatment of lowback pain. Tianjin $J$ Trad Chin Med. 2017;34(05):315-319.

46. Lai PH, Deng HH, Wu JM, et al. Clinical observation of electrothermic-needling moxibustion treating NLBP of cold-dampness pattern. J Clin Acupuncture Moxibustion. 2019;35 (01):30-33.

47. Li JH. Clinical observation on chronic nonspecific low back pain treated with oblique acupuncture. J Shanxi Univ Chin Med. 2017;18 (06):50-52.

48. Tian J, Niu J. Observation on the clinical effect of "Yi sui tong du" acupuncture on chronic non-specific low back pain. Clin J Med Officers. 2019;47(06):618-619.

49. Comachio J, Oliveira CC, Silva IFR, et al. Effectiveness of manual and electrical acupuncture for chronic non-specic low back pain: a randomized controlled trial. $J$ Acupunct Meridian Stud. 2020;13:87-93. doi:10.1016/j.jams.2020.03.064

50. Ushinohama A, Cunha BP, Costa LO, et al. Effect of a single session of ear acupuncture on pain intensity and postural control in individuals with chronic low back pain: a randomized controlled trial. Braz J Phys Ther. 2016;20(4):328-335. doi:10.1590/bjpt-rbf.2014.0158

51. Leite PMS, Mendonça ARC, Maciel LYS, et al. Does electroacupuncture treatment reduce pain and change quantitative sensory testing responses in patients with chronic nonspecific low back pain? A randomized controlled clinical trial. Evid Based Complement Alternat Med. 2018;2018:8586746. doi:10.1155/2018/8586746

52. Vas J, José MA, Modesto M, et al. Acupuncture in patients with acute low back pain: a multicentre randomized controlled clinical trial. Pain. 2012;153(9):1883-1889. doi:10.1016/j.pain.2012.05.033

53. Skonnord T, Skjeie H, Brekke M, et al. Acupuncture for acute nonspecific low back pain: a randomized, controlled, multicentre intervention study in general practice - the Acuback study. BMJ Open. 2020;10(8):e034157. doi:10.1136/bmjopen-2019-034157

54. Hasegawa TM, Baptista AS, de Souza MC, et al. Acupuncture for acute non-specific low back pain: a randomised, controlled, double-blind, placebo trial. Acupunct Med. 2014;32(2):109-115. doi:10.1136/acupmed-2013-010333

55. Cho YJ, Song YK, Cha YY, et al. Acupuncture for chronic low back pain: a multicenter, randomized, patient-assessor blind, sham-controlled clinical trial. Spine. 2013;38(7):549-557. doi:10.1097/BRS.0b013e318275e601

56. Chaimani A, Higgins JP, Mavridis D, Spyridonos P, Salanti G. Graphical tools for network meta-analysis in STATA. PLoS One. 2013.

57. French SD, Cameron M, Walker BF, et al. Superficial heat or cold for low back pain. Cochrane Database Syst Rev. 2006;1(1):CD004750.

58. Traeger AC, Hubscher M, Henschke N, et al. Effect of primary carebased education on reassurance in patients with acute low back pain. JAMA Intern Med. 2015;175(5):733-743. doi:10.1001/ jamainternmed.2015.0217

59. National Institute for Health and Care Excellence. Non-specific low back pain and sciatica: management. NICE guideline: short version. Draft for consultation. 2016. 
60. Zhang XX, Wu ZH, Dong MX. Preliminary study on curing mechanism of fire needle therapy. Beijing J Trad Chin Med. 2007;2007(09):576-578.

61. Zhang JY, Chen WD, Liu YR, et al. The origin and modern application of fire needle therapy. Asia Pac Trad Med. 2016;12(03):42-44.

62. Liberati A, Altman DG, Tetzlaff J, et al. The PRISMA statement for reporting systematic reviews and meta- analyses of studies that evaluate healthcare interventions: explanation and elaboration. BMJ. 2009;339:b2700. doi:10.1136/bmj.b2700
63. Zeng XT, Cao SY, Sun F, et al. Meta analysis series VI: indirect comparison and network analysis. Chin J Evid Based Cardiovasc Med. 2012;4(05):399-402.

\section{Publish your work in this journal}

The Journal of Pain Research is an international, peer reviewed, open access, online journal that welcomes laboratory and clinical findings in the fields of pain research and the prevention and management of pain. Original research, reviews, symposium reports, hypothesis formation and commentaries are all considered for publication. The manuscript management system is completely online and includes a very quick and fair peer-review system, which is all easy to use. Visit http:// www.dovepress.com/testimonials.php to read real quotes from published authors. 